\title{
Technological Advancements to Manage an Ancient Disease: Tracheal Stenosis
}

\section{Hans J. Lee* and Ray Wesley Shepherd}

Division of Pulmonary and Critical Care Medicine, Virginia Commonwealth University Medical Center, Richmond, VA 23298, USA

Tracheal stenosis (TS) represents an ancient medical disease that has transformed in its etiology and management with the advancement of technology. Pre-1930, TS was seen predominantly in pediatric diseases and infections such as diphtheria, syphilis, and tuberculosis [1]. Traumatic causes of TS became more common with the popularity of automobiles after the 1920's. Further technological advancement in the 1970's with mechanical ventilation made iatrogenic causes from prolonged intubation the most common cause of TS.

The development of endoscopic management of TS was initially slow, but grew rapidly with advancing technology. In 1897, Gustav Killian performed the first reported rigid bronchoscopy for a foreign body extraction [2]. It would be another century before Chevalier Jackson, developed rigid bronchoscopy and tools for TS in the 1920's [3]. Jackson refused to patent his tools to allow for widespread usage of a novel technology during his time. Another half a century would pass till Shigeto Ikeda developed the first flexible bronchoscope in 1966, which allowed for a more widespread method to diagnose TS. For the first time, non-surgeons were directly involved in the management of TS. The time interval for development of new techniques for endoscopic TS treatment would come in shorter intervals with the development of silicone tracheal stents by Jena Francois Dumon and the birth of a new field called, interventional pulmonology [4]. Over this past decade we have seen even more options available for the management of tracheal stenosis.

The advent of the field of Interventional Pulmonology has coincided with tracheal stenosis management. Specialized medical centers in interventional pulmonology have developed across the country offering endoscopic management of tracheal stenosis with a multi-disciplinary approach across specialties such as interventional pulmonology, thoracic surgery, radiology, anesthesia, and ENT. A disease once elusive with limited treatment options have become a disease with options including long term endoscopic treatment or endososcopic treatment as a bridge to various surgical options. In addition, the field of interventional pulmonology has brought clinical research and awareness to the main stream pulmonary community through its own development as a sub-specialty.

In this issue of Pulmonary and Respiratory Medicine, there are several articles discussing current endoscopic treatments for tracheal stenosis. While nobody can predict the future, developments in endoscopic treatment will be likely to occur in an even shorter time interval.

\section{References}

1. Santos D, Mitchell R (2010) The history of pediatric airway reconstruction. Laryngoscope 120: 815-820.

2. Zollner F (1965) Gustav Killian, father of bronchoscopy. Arch Otolaryngol 82 656-659.

3. Jackson CL (1942) Disease and management of the larynx. (2ndedn), NY Macmillan, New York, 202-207.

4. Dumon JF (1990) A dedicated tracheobronchial stent. Chest 97: 328-332.
*Corresponding author: Hans J. Lee, MD, Assistant Professor of Medicine, Virginia Commonwealth University Medical Center, PO Box 980050, Richmond VA 23298, USA, E-mail: hjlee@vcu.edu

Received March 27, 2012; Accepted March 29, 2012; Published April 01, 2012

Citation: Lee HJ, Shepherd RW (2012) Technological Advancements to Manage an Ancient Disease: Tracheal Stenosis. J Pulmonar Respirat Med 2:e116. doi:10.4172/2161-105X.1000e116

Copyright: @ 2012 Lee HJ. This is an open-access article distributed under the terms of the Creative Commons Attribution License, which permits unrestricted use, distribution, and reproduction in any medium, provided the original author and source are credited. 